\title{
Galectin-3, fibronectin-1, CITED-1, HBME1 and cytokeratin-19 immunohistochemistry is useful for the differential diagnosis of thyroid tumors
}

\author{
Manju L Prasad ${ }^{1}$, Natalia S Pellegata ${ }^{2}$, Ying Huang ${ }^{2}$, Haikady N Nagaraja ${ }^{3}$, \\ Albert de la Chapelle ${ }^{2}$ and Richard T Kloos ${ }^{4,5}$
}

${ }^{1}$ Department of Pathology; ${ }^{2}$ Human Cancer Genetics Program, Comprehensive Cancer Center; ${ }^{3}$ Department of Statistics; ${ }^{4}$ Division of Endocrinology, Diabetes, and Metabolism, Department of Internal Medicine and ${ }^{5}$ Division of Nuclear Medicine, Department of Radiology, The Ohio State University Medical center, Columbus, $\mathrm{OH}$, USA

\begin{abstract}
The diagnosis of thyroid tumors is critical for clinical management; however, tumors with follicular architecture often present problems. We evaluated the diagnostic use of the protein expression of four genes that were found to be upregulated in papillary thyroid carcinoma compared to normal thyroid (LGALS3, FN1, CITED1 and KRT19), and of the mesothelial cell surface protein recognized by monoclonal antibody HBME1 in thyroid tumors. Tissues from 85 carcinomas (67 papillary, six follicular, eight Hürthle cell and four anaplastic) and 21 adenomas were evaluated by immunohistochemistry for the expression of these gene protein products, for example, galectin-3 (GAL3), fibronectin-1 (FN1), CITED1, cytokeratin-19 (CK19) and HBME1. Non-neoplastic thyroids (29 adenomatous and 14 thyrotoxic hyperplasia, and 59 normal) were also studied. The expression of all five proteins was significantly associated with malignancy, and highly specific $(\geq 90 \%)$ for carcinoma compared to adenoma. GAL3, FN1 and/or HBME1 expression was seen in 100\% of carcinomas (85/85) and in $24 \%$ of adenomas (5/21). Coexpression of multiple proteins was seen in $95 \%$ of carcinomas and only $5 \%$ of adenomas $(P<0.0001)$. Coexpression of FN1 and GAL3 (FN1 + GAL3 +, 70/85) or FN1 and HBME1 (FN1 + HBME1 +, 53/85) was restricted to carcinomas, while their concurrent absence (FN1-GAL3 - or FN1-HBME1-, 18/21 adenoma) was highly specific $(96 \%)$ for benign lesions. Among non-neoplastic thyroids, adenomatous hyperplasia frequently expressed GAL3 $(n=16)$, CK19 $(n=9)$ and CITED1 $(n=7)$, but the expression was predominantly focal in contrast to the diffuse expression in carcinomas. An immunohistochemical panel consisting of GAL3, FN1 and HBME1 may be useful in the diagnosis of follicular cell-derived thyroid tumors.
\end{abstract}

Modern Pathology (2005) 18, 48-57, advance online publication, 23 July 2004; doi:10.1038/modpathol.3800235

Keywords: CITED1; cytokeratin; fibronectin; galectin; HBME1; immunohistochemistry; thyroid carcinoma

The prognosis and management of thyroid nodules depends on their diagnoses. The current diagnostic 'gold standard' is pathologic evaluation using routine hematoxylin and eosin (H\&E) stains. However, morphologic similarities between benign and

Correspondence: Dr ML Prasad, MD, Department of Pathology, The Ohio State University Medical Center, E 418 Doan Hall, 410 W 10th Ave., Columbus, OH 43210, USA.

E-mail: prasad-1@medctr.osu.edu

Abstract presented at the 92nd Annual Meeting of the United States and Canadian Academy of Pathology, Washington, DC, 2003.

Received 13 May 2004; revised and accepted 22 June 2004; published online 23 July 2004 malignant lesions are frequent, and follicular and papillary architectures may be seen in both benign and malignant lesions. Several critical features of malignancy, for example, pale nuclei for papillary thyroid carcinoma are open to subjective interpretations, and interobserver disagreements among pathologists are well documented. Saxen et al found only $58 \%$ agreement among five Nordic pathologists who tested the reproducibility of the WHO classification (1978) on 696 thyroid nodules. A more recent study compared the diagnoses of 21 follicular nodules by four American and four Japanese pathologists and showed an agreement of benign vs malignant in only $62 \%$ of the nodules. ${ }^{2}$ A review of 200 thyroid tumors by seven Italian 
pathologists revealed good agreement for papillary and anaplastic thyroid carcinomas, moderate for medullary and poor for follicular thyroid carcinomas. ${ }^{3}$ In another review of 41 follicular carcinomas by five experienced French thyroid pathologists, the agreement for malignancy varied from $5 \%$ among all five pathologists to $56 \%$ between two pathologists. ${ }^{4}$ Of concern, the interobserver and intraobserver agreements for vascular invasion were only 20 and $68 \%$, respectively. Thus, despite well-described criteria, the diagnostic agreement among pathologists remains poor. This underscores the need for additional diagnostic markers.

Recently, our group published a consistent gene expression profile for papillary thyroid carcinoma compared to normal thyroid. ${ }^{5}$ Here, we study the protein expression of four of the upregulated genes, LGALS3, FN1, CITED1 and KRT19, and the expression of the mesothelial cell surface protein HBME1 in various thyroid nodules to evaluate their usefulness in differential diagnosis. LGALS3 codes for galectin-3 (GAL3), a $\beta$-galactosil-binding lectin involved in regulating cell-cell and cell-matrix interactions. It is expressed in normal breast epithelial cells, inflammatory cells and various malignant cells. Several investigators have found GAL3 expression to be of value in discriminating between benign and malignant thyroid nodules. ${ }^{6-12}$ Fibronectin is an extracellular matrix protein produced by fibroblasts. Production of fibronectin by thyroid follicular cells is believed to be associated with transformation. ${ }^{5,13-15}$ Using the reverse transcription-PCR technique, Takano et $a l^{16}$ have suggested that fibronectin may be an accurate preoperative molecular diagnostic marker for papillary thyroid carcinoma. The CITED1 gene encodes a $27-\mathrm{kDa}$ protein belonging to the CITED (CBP/p300Interacting Transactivators with glutamic acid $[E]$ and aspartic acid $[D]$-rich C-terminal domain) family of nuclear proteins. ${ }^{17}$ The CITED proteins are believed to coregulate nuclear transcription proteins. Initial studies demonstrated a possible role in melanocyte differentiation, hence the synonym MSG1 (melanocyte-specific gene 1). ${ }^{18}$ Nuclear and cytoplasmic expression of CITED1 has been noted in melanocytes, breast epithelial cells, and testicular germ cells. KRT19 encodes cytokeratin-19 (CK19), a cytoskeletal protein that is significantly increased in papillary thyroid carcinoma, and has been reported to be helpful in distinguishing it from other benign and malignant thyroid nodules. ${ }^{5,19-23}$ HBME1, a monoclonal antibody generated against a suspension of malignant epithelial mesothelioma cells, reacts with the microvillous surface protein of mesothelial cells. We included HBME1 because its expression has been reported in papillary and follicular thyroid carcinoma but not in normal thyroid cells. ${ }^{23-25}$

\section{Materials and methods}

The study was comprised of 215 formalin-fixed paraffin-embedded thyroid tissues (Table 1). There were 67 papillary thyroid carcinoma, six follicular, eight Hürthle cell and four anaplastic carcinomas. The papillary thyroid carcinomas included 44 classic, 13 follicular, seven oncocytic, one columnar and two solid/poorly differentiated variants. Follicular and Hürthle cell carcinomas were diagnosed by the presence of complete capsular and/or vascular invasion, and the absence of nuclear features of papillary thyroid carcinoma. Hürthle cell carcinomas were composed of greater than $75 \%$ oncocytic cells having moderate to abundant eosinophilic granular cytoplasm. ${ }^{26}$ Anaplastic thyroid carcinomas were comprised of high-grade pleomorphic spindle cells. Adenomas were defined as completely encapsulated follicular or Hürthle cell tumors with homogeneous architecture and morphology, lacking nuclear features of papillary thyroid carcinoma and without capsular and vascular invasion. Encapsulated follicular tumors showing atypical architecture (solid/trabecular pattern),

Table 1 Protein expression in thyroid

\begin{tabular}{|c|c|c|c|c|c|c|}
\hline Diagnosis & GAL3 & FN1 & CITED1 & $H B M E 1$ & CK19 & Coexpression $^{\mathrm{a}}$ \\
\hline \multicolumn{7}{|l|}{ Carcinoma } \\
\hline Papillary $(n=67)$ & $63(94 \%)$ & $61(91 \%)$ & $58(87 \%)$ & $57(85 \%)$ & $48(72 \%)$ & $65(97 \%)$ \\
\hline Follicular $(n=6)$ & $4(66 \%)$ & $3(50 \%)$ & $3(50 \%)$ & $3(50 \%)$ & $3(50 \%)$ & $5(83 \%)$ \\
\hline Hürthle cell $(n=8)$ & $7(88 \%)$ & $6(75 \%)$ & $2(25 \%)$ & $1(13 \%)$ & $4(50 \%)$ & $7(88 \%)$ \\
\hline Anaplastic $(n=4)$ & $4(100 \%)$ & $4(100 \%)$ & $0(0 \%)$ & $0(0 \%)$ & $1(25 \%)$ & $4(100 \%)$ \\
\hline Neoplasms of undetermined malignant potential $(n=7)$ & $6(86 \%)$ & $1(14 \%)$ & $3(43 \%)$ & $2(29 \%)$ & $1(14 \%)$ & $3(43 \%)$ \\
\hline Adenoma $(n=21)$ & $2(10 \%)$ & $1(5 \%)$ & $2(10 \%)$ & $2(10 \%)$ & $1(5 \%)$ & $1(5 \%)$ \\
\hline \multicolumn{7}{|l|}{ Non-neoplastic thyroid } \\
\hline Nodular goiter $(n=29)$ & $16(55 \%)$ & $2(7 \%)$ & $7(24 \%)$ & $1(3 \%)$ & $9(31 \%)$ & $11(38 \%)$ \\
\hline Thyrotoxic hyperplasia $(n=14)$ & $1(7 \%)$ & $0(0 \%)$ & $0(0 \%)$ & $0(0 \%)$ & $0(0 \%)$ & $0(0 \%)$ \\
\hline Normal $(n=59)$ & $0(0 \%)$ & $0(0 \%)$ & $0(0 \%)$ & $0(0 \%)$ & $4(7 \%)$ & $0(0 \%)$ \\
\hline
\end{tabular}

GAL3 and/or FN1 and/or HBME1: 85/85 carcinoma and 5/21 adenoma.

${ }^{\mathrm{a}}$ Coexpression of $\geq 2$ proteins. 
Table 2 Antibodies used in this study

\begin{tabular}{|c|c|c|c|}
\hline Antibody & Clone $\&$ source & Dilution & Source \\
\hline GAL3 & Monoclonal, NCL-Gal3 & $1: 300$ & Novocastra, UK \\
\hline HBME1 & Monoclonal, HBME-1 & $1: 200$ & Dako, Carpinteria, CA, USA \\
\hline CK19 & Monoclonal, RCK108 & $1: 100$ & Dako, Carpinteria, CA, USA \\
\hline FN1 & Polyclonal, A0245 & $1: 2000$ & Dako, Carpinteria, CA, USA \\
\hline CITED1 & Polyclonal, J72220K & $1: 2000$ & T Shioda, Boston, MA, USA \\
\hline
\end{tabular}

Dako's Target Retrieval Solution was used to carry out the heat-induced antigen retrieval for GAL3, FN1 and CITED1.

severe cytologic atypia or incomplete capsular invasion without definite vascular invasion were classified as follicular neoplasms of uncertain malignant potential. ${ }^{27,28}$ We also included 29 nodular goiters, 14 diffuse thyrotoxic hyperplasia and 59 normal thyroid tissues to study the protein expression in non-neoplastic thyroid lesions. All cases were initially diagnosed by a pathologist (not a coauthor) and then reviewed by a second pathologist (MLP).

Immunohistochemistry was performed on $4 \mu \mathrm{m}$ thick sections using the labeled streptavidin-biotin peroxidase complex system (LSAB2) in a Dako Autostainer (DakoCytomation, Carpinteria, CA, USA). Heat-induced antigen retrieval was carried out for GAL3, FN1 and CITED1 (Dako's Target Retrieval solution, $\mathrm{pH}$ 6.1, steaming for $30^{\prime}$ at $94^{\circ} \mathrm{C}$ ) and sections were incubated with primary antibodies (Table 2) for $30^{\prime}-60^{\prime}$ at room temperature. After primary antibodies, all sections were blocked for endogenous avidin and biotin by incubating with avidin solution for $20^{\prime}$ followed by biotin solution for 20' (Dako's avidin/biotin blocking system, X0590). Positive controls were normal breast for GAL3 and CITED1, tonsil for FN1, small intestinal mucosa for CK19 and an epithelioid mesothelioma for HBME1. Appropriate negative controls by substituting primary antibody with isotype-matched mouse or rabbit IgG were also included.

\section{Interpretation and Analysis}

CITED1 and GAL3 expressions were nuclear and cytoplasmic. CK19 expression was cytoplasmic only. HBME1 and FN1 expressions were cytoplasmic and membranous with luminal accentuation. Normal FN1 expression in fibrous tissue and basement membrane served as internal control. The protein expression was initially assessed along a scale of $0-100 \%$ of tumor cells showing immunoreactivity. After evaluating normal and hyperplastic thyroid tissues, positive (increased) expression was defined as presence of $\geq 10 \%$ immunoreactive thyrocytes. Sensitivity (true positive/true positive + false negative), specificity (true negative/true negative + false positive) and accuracy (true positive + true negative/all positive + all negative) of the markers and their combinations were compared.
Two-sided Fisher's exact test was used to determine statistical significance with $\alpha$ level set at $\leq 0.05$.

\section{Results}

The expression of GAL3, FN1, CITED1, HBME1 and CK19 in various thyroid tissues is shown in Table 1 (Figures 1-3). In general, the expression of the proteins in malignant tumors was diffuse, while in benign tumors and non-neoplastic thyroid tissues, there was no expression or focal expression, usually in less than a third of the tumor cells.

\section{Carcinomas}

Of 85 carcinomas, 81 (95\%) coexpressed multiple proteins (Tables 1 and 3, Figures 1 and 2). There was no significant difference in protein expression among the variants of papillary thyroid carcinoma. All anaplastic thyroid carcinomas diffusely and strongly expressed GAL3 and FN1 (100\%), and one additionally showed focal CK19 expression (Figure 2 ). The expression was more frequently diffuse in papillary and anaplastic carcinomas compared to others.

\section{Adenomas \\ There were 19 follicular and two Hürthle cell adenomas. Two adenomas showed expression of GAL3 in approximately 10\% of tumor cells (Figure 3 ). One adenoma each showed diffuse expression of FN1, CITED1, HBME1 and CK19, while a single adenoma (5\%) coexpressed two proteins (CITED1+ HBME1 +). Hürthle cell adenomas did not express any of the proteins.}

\section{Neoplasms of Uncertain Malignant Potential}

There were six follicular and one Hürthle cell tumor of undetermined malignant potential. All tumors were encapsulated follicular neoplasms showing incomplete capsular invasion $(n=5)$ and/or some nuclear atypia that did not meet the criteria for papillary thyroid carcinoma $(n=3)$. The expression of proteins was usually focal with diffuse expression 

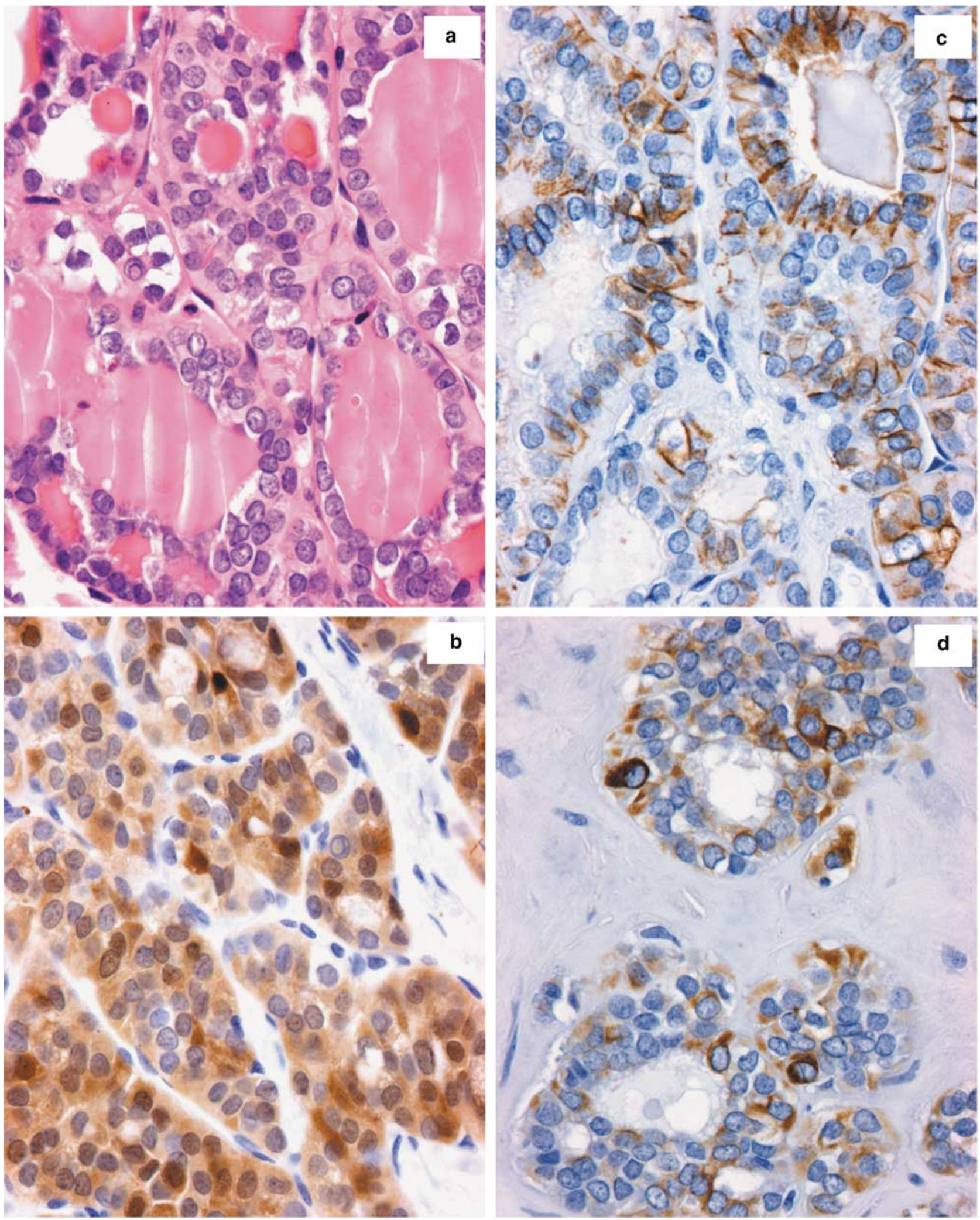

Figure 1 (a) Follicular variant of papillary thyroid carcinoma showing expression of (b) CITED1, (c) HBME1 and (d) CK19.

of GAL3 and CITED1 being seen in two tumors each. Three tumors $(43 \%)$ showed coexpression of multiple proteins (GAL3 + HBME1 + CITED1 + in two and GAL3 + FN1 + CITED1 + CK19 + in one tumor). No correlation was noted between their morphology and the protein expression. 

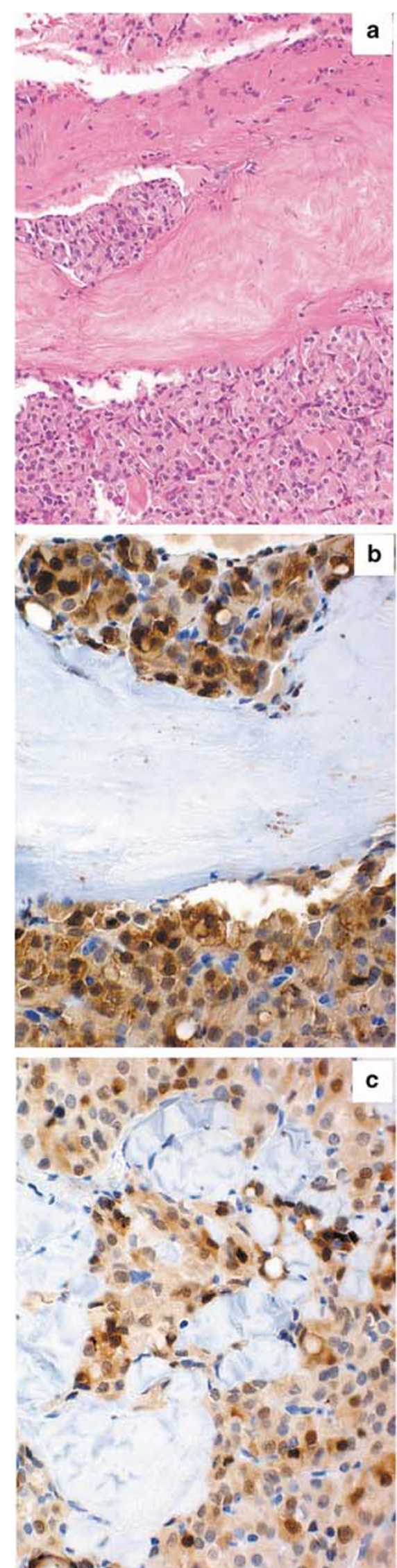
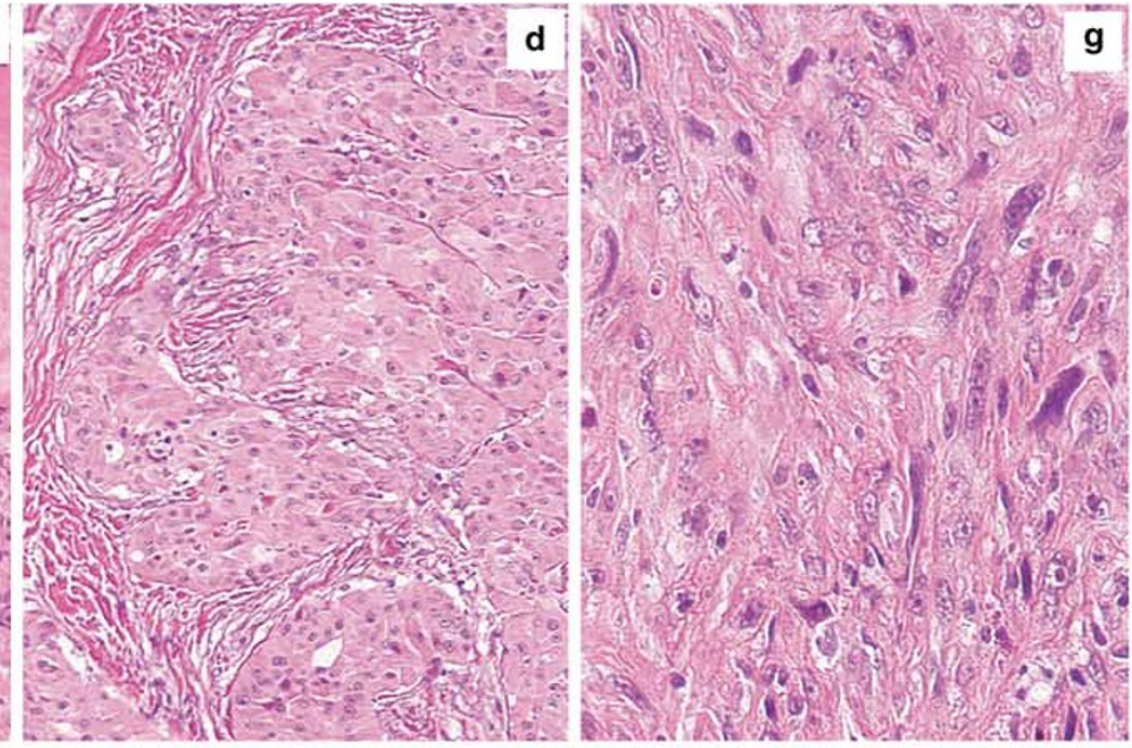

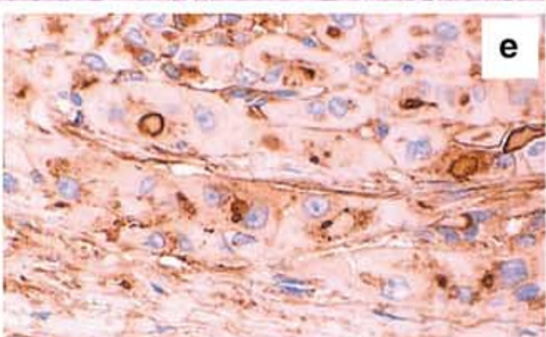

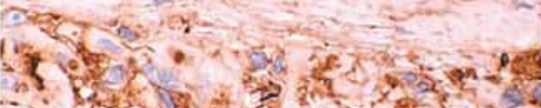

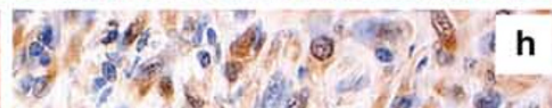

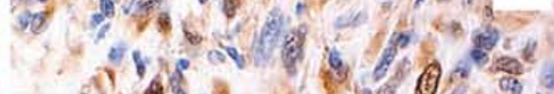
a) of $0^{\circ}$. s. $10 \%$ -

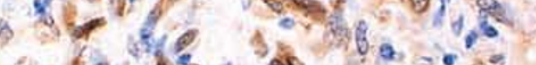

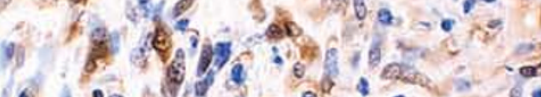

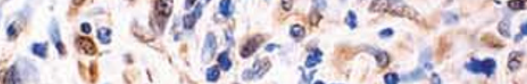

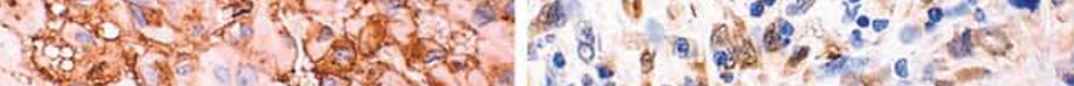

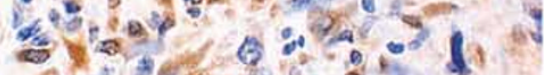

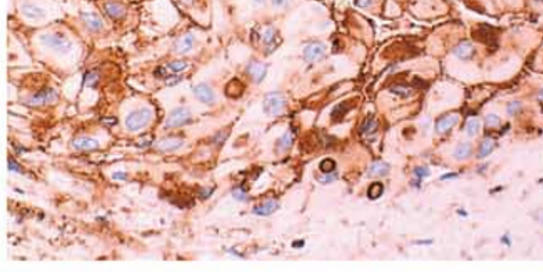

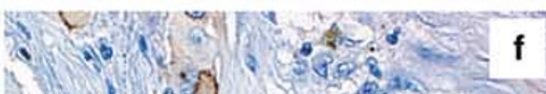

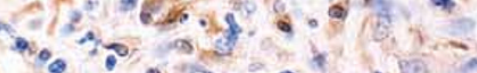

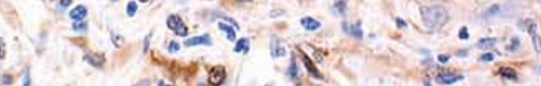
y tor of of

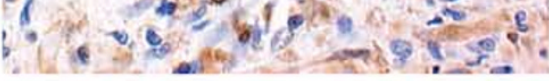

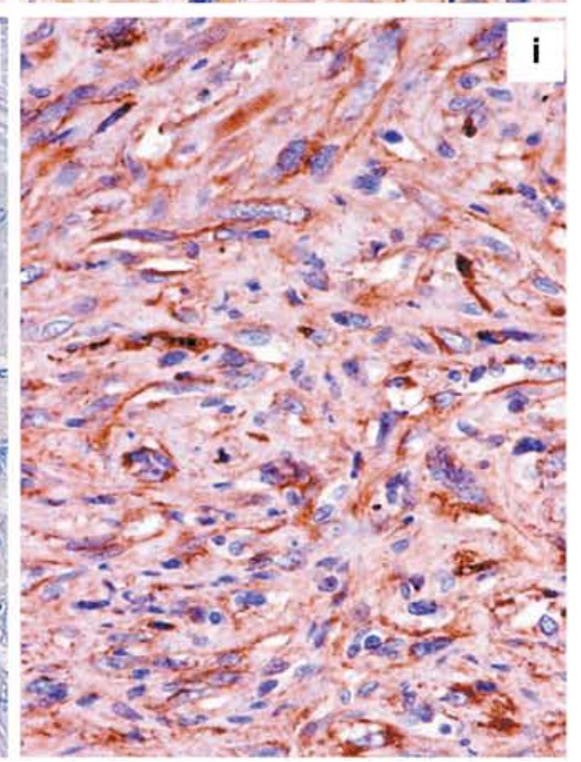




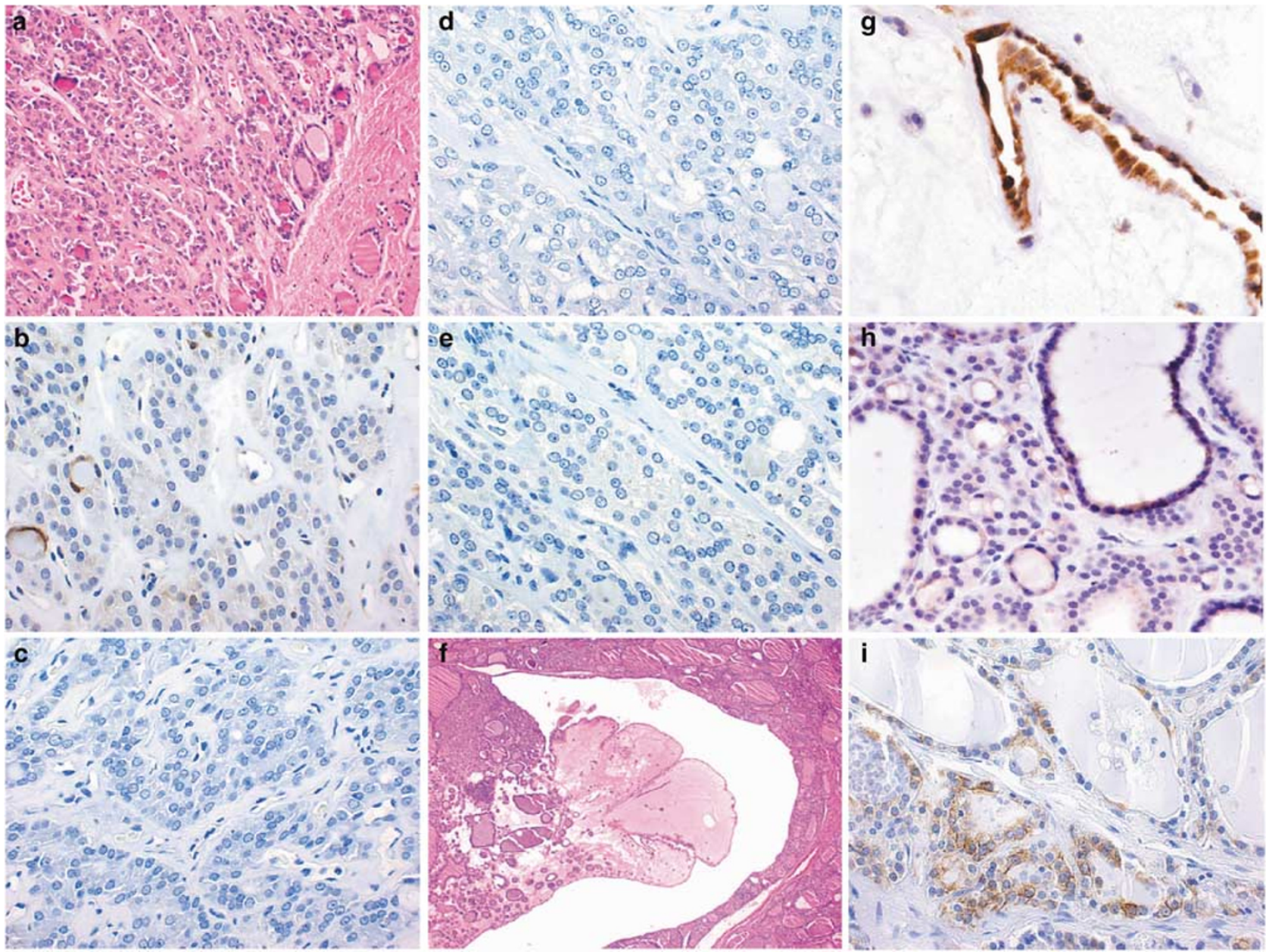

Figure 3 Expression of proteins in benign thyroid nodules: (a) Follicular adenoma showing (b) focal GAL3 expression, but (c) no expression of FN1, (d) HBME1, and (e) CITED1. (f) Nodular goiter with cystic degeneration and papillary hyperplasia showing (g) GAL3, (h) weak CITED1, and (i) CK19 expression.

\section{Non-Neoplastic Thyroids}

Nodular goiters frequently showed follicular and focal papillary hyperplasia associated with fibrosis, hemorrhage, inflammation and cystic degeneration, and in addition to thyrocytes, several other cells expressed one or more proteins. Fibroblasts and inflammatory cells including macrophages expressed GAL3. Solid cell nests and metaplastic squamous cells expressed GAL3 and CK19. GAL3 was the most frequently expressed protein (55\%) followed by CK19 (31\%) and CITED1 (24\%) in the thyrocytes (Table 1, Figure 3). However, the protein expression was usually focal with only three goiters $(10 \%)$ showing diffuse expression of GAL3 and one each $(3 \%)$ showing diffuse expression of CK19 and CITED1. FN1 expression was observed in extracellular fibrosis in most goiters but was considered positive in only two cases that demonstrated cytoplasmic and membranous expression of FN1 in thyrocytes in association with fresh hemorrhage and fibrin deposition. Coexpression of multiple proteins, usually GAL3 and/or CK19 and/or CITED1 was seen in 11 goiters (38\%).

All 14 diffuse thyrotoxic hyperplasia showed focal or diffuse papillary hyperplasia and did not express any protein except one case that focally expressed GAL3 in approximately $10 \%$ of cells. Four normal thyroid tissues focally expressed CK19 in approximately $10-20 \%$ of thyrocytes. Normal thyroids did not express any other proteins. Thyrotoxic hyperplasia and normal thyroid tissues did not show diffuse expression or coexpression of proteins.

\section{Statistical Analysis}

The significance of these markers in differentiating malignant from benign tumors is shown in Tables 3

Figure 2 Expression of proteins in thyroid carcinomas: (a) Follicular thyroid carcinoma showing vascular invasion in a capsular blood vessels, (b) GAL3 expression in the tumor and the intravascular tumor emboli, and (c) CITED1 expression in tumor cells invading capsule. (d) Hürthle cell carcinoma showing (e) diffuse FN1 and (f) focal HBME1 expression. (g) Anaplastic thyroid carcinoma showing diffuse (h) GAL3 and (i) FN1 expression in malignant spindle cells. 
Table 3 Coexpression and concurrent absence of expression

\begin{tabular}{|c|c|c|c|c|}
\hline Coexpression & $\begin{array}{c}\text { Carcinoma } \\
(n=85)\end{array}$ & $\begin{array}{c}\text { Adenoma } \\
(n=21)\end{array}$ & $\begin{array}{l}\text { Sensitivity } \\
\text { for } \\
\text { carcinoma } \\
\quad \%)\end{array}$ & $\begin{array}{l}\text { Specificity } \\
\text { for } \\
\text { carcinoma } \\
(\%)\end{array}$ \\
\hline FN1+GAL3+ & 70 & $0(0 \%)$ & 82 & 100 \\
\hline FN1+HBME1+ & 53 & $0(0 \%)$ & 62 & 100 \\
\hline $\begin{array}{l}>\text { any two } \\
\text { proteins }^{\mathrm{a}}\end{array}$ & 81 & $1(5 \%)$ & 95 & 95 \\
\hline $\begin{array}{l}\text { Concurrent } \\
\text { absence }\end{array}$ & & & $\begin{array}{l}\text { Sensitivity } \\
\text { for } \\
\text { adenoma } \\
(\%)\end{array}$ & $\begin{array}{c}\text { Specificity } \\
\text { for } \\
\text { adenoma } \\
(\%)\end{array}$ \\
\hline FN1- GAL3- ${ }^{\mathrm{b}}$ & $3(4 \%)$ & 18 & 86 & 96 \\
\hline FN1-HBME1- ${ }^{\mathrm{b}}$ & $3(4 \%)$ & 18 & 86 & 96 \\
\hline
\end{tabular}

FN1+GAL3+: 59 papillary, two follicular, five Hürthle cell and four anaplastic carcinoma.

FN1+HBME1+: 52 papillary and one follicular carcinoma

FN1-GAL3-: two papillary and one follicular carcinoma.

FN1-HBME1-: one papillary, one follicular and one Hurthle cell carcinoma.

${ }^{\mathrm{a}} P<0.0001$, accuracy for carcinoma- $-95 \%$.

${ }^{\mathrm{b}}$ Accuracy for adenoma-94\%.

Table 4 Differential diagnosis of benign and malignant tumors with follicular architecture

\begin{tabular}{lccc}
\hline Proteins & $\begin{array}{c}\text { Carcinoma } \\
n=28 \\
\text { (sensitivity) }\end{array}$ & $\begin{array}{c}\text { Adenoma } \\
n=21\end{array}$ & $\begin{array}{c}\text { Specificity } \\
(\%)\end{array}$ \\
\hline GAL3 $^{\text {b }}$ & $25(89 \%)$ & $2(10 \%)$ & 90 \\
FN1 & $21(75 \%)$ & $1(5 \%)$ & 95 \\
CITED1 & $19(68 \%)$ & $2(10 \%)$ & 90 \\
CK19 & $18(64 \%)$ & $1(5 \%)$ & 95 \\
HBME1 & $17(61 \%)$ & $2(10 \%)$ & 90 \\
\hline
\end{tabular}

${ }^{\mathrm{a}}$ Follicular variants of 14 papillary carcinoma, six follicular carcinoma and eight Hürthle cell carcinoma.

${ }^{\mathrm{b}}$ Accuracy of GAL3 was the highest $(90 \%)$.

and 4 and Figure 4. All five proteins were highly specific ( $\geq 90 \%$ ) for carcinomas. GAL3 was the most sensitive but failed to detect seven carcinomas $(8 \%$ false negative). Tables 1 and 3 show that coexpression of multiple proteins was significantly more frequent in carcinomas than adenomas $(P<0.0001)$. Coexpression of some proteins, for example, FN1 + GAL3 + or FN1 + HBME1 + immunophenotype was observed only in carcinomas (100\% specific), while their concurrent absence, that is, FN1-GAL3- or FN1-HBME1 - immunophenotype was highly specific (96\%) for adenomas (Table 3). Table 4 compares the expression of these proteins in benign and malignant tumors with follicular architecture, that is, 21 adenomas vs 14 follicular variants of papillary carcinoma, six follicular and eight Hürthle cell carcinomas. All five markers were highly specific $(\geq 90 \%)$ for carcinoma and GAL3 was the most sensitive $(89 \%)$ and accurate $(90 \%)$. CITED1 and/or

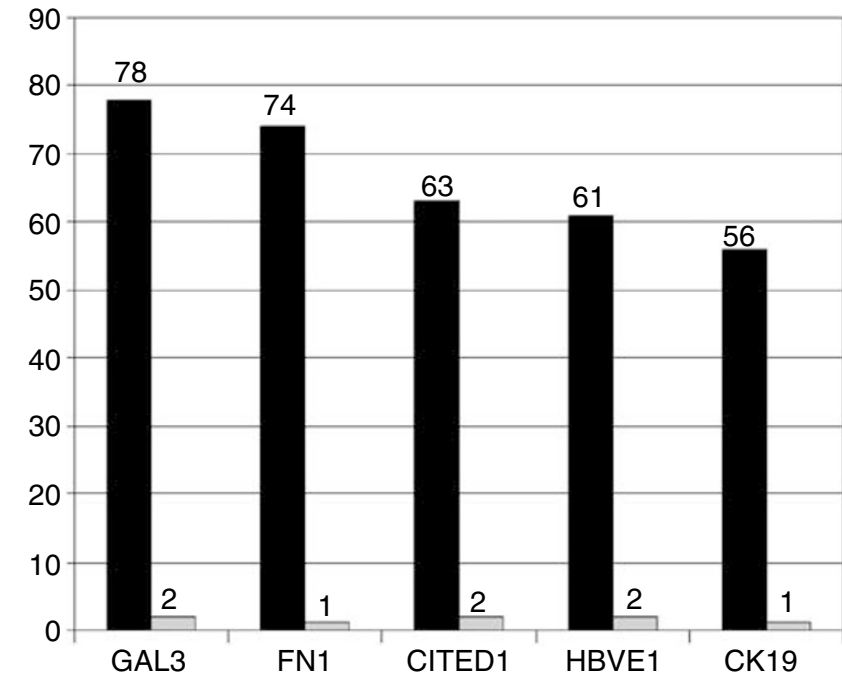

Figure 4 Differential expression of proteins in 85 carcinomas and 21 adenomas. GAL3: sensitivity $92 \%$, specificity 90\%, FN1: sensitivity $87 \%$, specificity $95 \%$, CITED1: sensitivity $74 \%$, specificity 90\%, HBME1: sensitivity $72 \%$, specificity $90 \%$, CK19: sensitivity $66 \%$, specificity $95 \%$.

Table 5 Follicular variant of papillary carcinoma vs follicular and Hürthle cell carcinoma

\begin{tabular}{llcl}
\hline Protein & $\begin{array}{c}\text { Papillary } \\
\text { carcinoma } \\
(n=14)\end{array}$ & $\begin{array}{c}\text { Follicular+ } \\
\text { Hürthle cell } \\
\text { carcinoma } \\
(n=14)\end{array}$ & P-value \\
\hline CITED1 & $\mathbf{1 4}(\mathbf{1 0 0} \%)$ & $\mathbf{5 ( 3 6 \% )}$ & $\leq \mathbf{0 . 0 0 1}$ \\
HBME1 & $\mathbf{1 3}(\mathbf{9 3} \%)$ & $\mathbf{4 ( 2 9 \% )}$ & $\leq \mathbf{0 . 0 1}$ \\
CITED1+HBME1 & $\mathbf{1 3}(\mathbf{9 3} \%)$ & $\mathbf{2 ( 1 4 \% )}$ & $<\mathbf{0 . 0 0 0 1}$ \\
GAL3 & $14(100 \%)$ & $11(79 \%)$ & 0.22 \\
FN1 & $12(86 \%)$ & $9(64 \%)$ & 0.38 \\
CK19 & $11(79 \%)$ & $7(50 \%)$ & 0.23 \\
& & &
\end{tabular}

HBME1 were significantly more associated with papillary than follicular or Hürthle cell carcinoma $(P<0.01$, Table 5).

GAL3, FN1 and/or HBME1 were expressed in $100 \%$ of carcinoma, $86 \%$ of tumors of undetermined malignant potential (6/7) and $24 \%$ adenomas (5/21). GAL3, FN1 and/or CITED1 also revealed similar frequency of expression. All other triple or double antibody combinations were less than $100 \%$ sensitive for carcinoma.

When the differentiation of carcinoma from benign nodules included goiters, GAL3 was the least specific followed by CK19 and CITED1 (55, 31 and $24 \%$ false-positive goiters, respectively), whereas HBME1 and FN1 were most specific (3 and $7 \%$ false-positive goiters, respectively).

The thyroid tumors of undetermined malignant potential showed expression profiles intermediate between adenomas and carcinomas. Three tumors showed coexpression of multiple proteins (43 vs 5\% adenoma vs $95 \%$ carcinoma). One tumor showed 
coexpression of FN1 + GAL3 + (14 vs 0\% adenoma vs $82 \%$ carcinoma), but none showed coexpression of $\mathrm{FN} 1+\mathrm{HBME} 1+\left(\begin{array}{l}0 \\ \text { vs }\end{array}\right.$ carcinoma). One tumor of undetermined malignant potential lacked expression of FN1 and GAL3 (14 vs $86 \%$ adenoma vs $4 \%$ carcinoma), and four tumors lacked expression of FN1 and HBME1 (57 vs 86\% adenoma vs $4 \%$ carcinoma).

\section{Discussion}

We selected four genes from among several genes found to be consistently upregulated in papillary thyroid carcinoma compared to normal thyroid because of the availability of antibodies against their protein products that gave robust immunoreaction in formalin-fixed paraffin-embedded tissues allowing their evaluation in archival tissues. The monoclonal antibody HBME1 was included for comparison because of its reported specificity for papillary thyroid carcinoma. ${ }^{23-25}$ Our study shows that these proteins were not only expressed in papillary thyroid carcinomas but also in other follicular cell-derived thyroid carcinomas. Indeed, this relative lack of specificity for malignant tumors was very useful in differentiating all carcinomas from adenomas.

One of the most frequent difficulties in thyroid pathology is differentiating adenomas from carcinomas, especially those with follicular architecture, for example, follicular variant of papillary thyroid carcinoma, follicular and Hürthle cell carcinomas. ${ }^{2,4}$ This differentiation is critical for the treatment and long-term management of the tumors. We found that all five markers were highly specific for carcinoma, but GAL3 was the most sensitive and accurate. Only two adenomas showed focal expression of GAL3 in approximately $10 \%$ of tumor cells. Other investigators have used GAL3 in differentiating follicular carcinomas from adenomas in fine-needle aspirates. ${ }^{9,11}$ However, certain problems emerged with GAL3. First, GAL3 expression was also seen in several nonthyroidal cells, for example, squamous cells, fibroblasts and inflammatory cells including macrophages. These nonthyroidal cells were often associated with hemorrhage, fibrosis and cystic degeneration, which are frequently present in nodular goiter. Indeed, GAL3 expression was seen in $55 \%$ of the goiters in the current series making it the least-specific marker if the differential diagnosis included goiters. Other investigators have also reported GAL3 expression in benign thyroid lesions by various techniques including reverse transcription-PCR. ${ }^{22,29-32}$ The second problem with GAL3 was that seven carcinomas $(8 \%)$ were false negative.

Therefore, we next attempted to identify a combination of markers with no false-negative results. We found that all carcinomas were positive for GAL3, FN1 and/or HBME1. Thus, an immunohistochemical diagnostic panel comprising of these three markers may improve sensitivity for carcinomas. This panel had other advantages as well, for example, coexpression of FN1 and GAL3 or FN1 and HBME1 (FN1 + GAL3 + or FN1 + HBME1 + phenotype) was diagnostic of carcinoma, whereas their concurrent absence (FN1-GAL3- or FN1-HBME1phenotype) was highly specific for benign lesions. FN1 was first reported to be overexpressed in thyroid tumors by immunohistochemistry in 1988, but we are unaware of any subsequent studies comparing it to other markers of thyroid malignancy or evaluating its expression in hyperplastic nodules. ${ }^{14}$ We found FN1 expression by tumor cells to be nearly as sensitive as GAL3, and highly specific for all follicular cell-derived thyroid carcinomas. Several recent studies have reported HBME1 expression to be diagnostically useful in papillary thyroid carcinoma. ${ }^{23,25}$ However, to the best of our knowledge, no single marker by itself is $100 \%$ sensitive for malignancy. By immunohistochemistry, ret/PTC and PPAR $\gamma$ are reported in $<70 \%$ of papillary and follicular thyroid carcinomas, respectively, while CD10 is expressed in follicular carcinoma and follicular variant of papillary carcinoma, but not in conventional papillary thyroid carcinoma. ${ }^{3-36}$ Therefore, a combination of multiple markers may be more sensitive than any single marker. Our study shows that a diagnostic immunohistochemical panel comprising of GAL3, FN1 and HBME1 was $100 \%$ sensitive for all follicular cellderived carcinomas. Although GAL3, FN1 and/or CITED1 were also expressed in all carcinomas, we preferred not to include CITED1 due to its higher false-positive results in goiters compared to FN1 and HBME1. In addition, unlike CITED1, antibodies to GAL3, FN1 and HBME1 are commercially available and can be readily used. No other combination of two or three proteins in our study proved to be $100 \%$ sensitive. HBME1 and CITED1 were highly specific for papillary thyroid carcinoma, especially when concurrently expressed, and were helpful in differentiating follicular variant of papillary from follicular and Hürthle cell carcinomas. Although this differentiation may not be critical for treatment, the precise classification of carcinoma is helpful in predicting outcome, and the route of metastasis. The association of HBME1 with papillary thyroid carcinoma in the current series is consistent with the observations made by other investigators. ${ }^{23,25}$

The current study included seven tumors classified as neoplasms of uncertain malignant potential, a concept initially proposed by Rosai et $a 2^{27}$ and later recommended by Williams ${ }^{28}$ following his examination of thyroid nodules that developed in the exposed population of the Chernobyl accident. The tumors were encapsulated, had a follicular architecture, did not show definite capsular or vascular invasion or showed some nuclear atypia and pallor that did not quite meet the diagnostic criteria for papillary thyroid carcinoma. Their 
protein expression pattern was intermediate between adenoma and carcinoma. Interestingly, GAL3 expression was seen in six of seven neoplasms of uncertain malignant potential, which may support the proposal made by some investigators that GAL3 is a marker of early malignant transformation and minimally invasive carcinoma. ${ }^{11,22}$ Our results suggest the 'indeterminate or borderline' nature of these lesions. Although these markers fail to classify these tumors as clearly malignant or benign, they may help in selecting tumors for prolonged follow-up in order to determine their true biologic nature.

The role of CK19 in the diagnosis of thyroid carcinoma has been controversial. This may be partially due to the subjectivity involved in assessing positive expression. Sahoo et $a^{21}$ found CK19 expression in all benign tumors although the majority of them expressed CK19 in $<5 \%$ of tumor cells. Other investigators have reported diffuse expression in papillary thyroid carcinoma compared to focal expression in other tumors and nodular goiters. ${ }^{19,20}$ Although we used $\geq 10 \%$ immunoreactive cells as the criteria for protein overexpression, we agree that sometimes benign and malignant tumors may show only borderline positive or negative reactions. Despite its apparent shortcoming, $\geq 10 \%$ was a useful cutoff value in differentiating carcinomas from adenomas.

Lack of expression of the papillary thyroid carcinoma-associated proteins in normal thyroid and diffuse thyrotoxic hyperplasia shows that their expression is not associated with papillary formation or hyperplasia. Interestingly, nodular goiters expressed several of these proteins, in thyrocytes and in other reactive cells. GAL3, CK19 and CITED1 expression was noted in 55, 31 and $24 \%$ of goiters respectively. GAL3 was expressed in squamous cells, proliferating fibroblasts and inflammatory cells, for example, macrophages. Metaplastic squamous cells in benign thyroid and the embryonic remnant ultimobranchial body expressed CK19, as has been also reported by others. ${ }^{23}$ Although only two goiters showed FN1 expression by thyrocytes, FN1 (being an extracellular matrix protein synthesized by fibroblasts) was increased in hemorrhage and fibrosis, often associated with goiters. Using $\geq 10 \%$ immunoreactive thyrocytes as the criteria for protein overexpression helped avoid overinterpretation of nonspecific immunoreactivity. False-positive protein expression in benign lesions was usually focal and in less than one-third of the thyrocytes. Multiple protein markers in conjunction with careful morphologic examination would help resolve problems associated with borderline expressions.

A limitation of all thyroid studies is that the 'gold standard' (H\&E examination) used to classify thyroid neoplasms as benign or malignant is flawed. Approximately half of our carcinomas had demonstrated metastases, and there was no difference in the immunohistochemical profile of those with metastases or without metastasis. To the best of our knowledge, none of the adenomas or tumors of 'undetermined malignant potential' have metastasized (data not shown). While presence of metastasis confirms malignancy, lack of metastasis does not rule it out. Thus, benign lesions may be difficult to differentiate from malignant tumors that did not metastasize and may have been cured by treatment. In the current study, all thyroid lesions were initially diagnosed by a staff pathologist and then reviewed by one of the authors (MLP). We made an attempt to include unequivocal cases, reserving the term 'undetermined malignant potential' for tumors that could not be definitively categorize as benign or malignant.

In conclusion, GAL3, FN1, HBME1, CITED1 and CK19 were expressed in all follicular cell-derived carcinomas. Their expression was helpful in differentiating adenomas from carcinomas, especially those with follicular architecture, that is, follicular variants of papillary carcinoma, follicular carcinoma and Hürthle cell carcinoma. An immunohistochemical panel consisting of GAL3, FN1 and HBME1 was $100 \%$ sensitive for carcinomas. The phenotype $\mathrm{FN} 1$ + GAL3 + or FN1 + HBME1 + was diagnostic of carcinoma, while the phenotype FN1-GAL3- or FN1-HBME1 - was highly specific of adenoma. CITED1 and HBME1 were significantly associated with papillary thyroid carcinoma and were helpful in differentiating follicular variants of papillary carcinoma from follicular carcinoma and Hürthle cell carcinoma. However, many of the proteins, for example, GAL3, CITED1 and CK19 were expressed focally in goiters. These markers need to be used in combination with one another, and in conjunction with careful morphologic evaluation.

\section{Acknowledgements}

We thank Dr Toshi Shioda, MD, PhD, Mass General Hospital Cancer Center, Boston, MA, for providing the anti-CITED1 antibody, Drs Saul Suster and Maria D Perez-Montiel for contributing cases to this series and Ms Susie Jones for technical assistance. This study was funded by the James Cancer Hospital \& Solove Research Institute, Comprehensive Cancer Center, Ohio State University, Columbus, Ohio, core Grant CA 16058, and NIH (NCRR) Grant M01RR00034.

\section{References}

1 Saxen E, Franssila K, Bjarnason O, et al. Observer variation in histologic classification of thyroid cancer. Acta Pathol Microbiol Scand 1978;86:483-486.

2 Hirokawa M, Carney JA, Goellner JR, et al. Observer variation in encapsulated follicular lesions of the thyroid. Am J Surg Pathol 2002;26:1508-1514. 
3 Fassina AS, Montesco MC, Ninfo V, et al. Histological evaluation of thyroid carcinomas: reproducibility of the 'WHO' classification. Tumori 1993;79:314-320.

4 Franc B. Observer variation of lesions of the thyroid. Am J Surg Pathol 2003;27:1177-1178.

5 Huang Y, Prasad M, Lemon WJ, et al. Gene expression in papillary thyroid carcinoma reveals highly consistent profiles. Proc Natl Acad Sci USA 2001;98:1504415049.

6 Chiariotti L, Berlingieri MT, De Rosa P, et al. Increased expression of the negative growth factor, galactosidebinding protein gene in transformed thyroid cells and in human thyroid carcinomas. Oncogene 1992;7:2507-2511.

7 Fernandez PL, Merino MJ, Gomez M, et al. Galectin-3 and laminin expression in neoplastic and non-neoplastic thyroid tissue. J Pathol 1997;181:80-86.

8 Gasbarri A, Martegani MP, Del Prete F, et al. Galectin-3 and CD44v6 isoforms in the preoperative evaluation of thyroid nodules. J Clin Oncol 1999;17:3494-3502.

9 Inohara $\mathrm{H}$, Honjo $\mathrm{Y}$, Yoshii $\mathrm{T}$, et al. Expression of galectin-3 in fine needle aspirates as a diagnostic marker differentiating benign from malignant thyroid neoplasms. Cancer 1999;85:2475-2484.

10 Orlandi F, Saggiorato E, Pivano G, et al. Galectin-3 is a presurgical marker of human thyroid carcinoma. Cancer Res 1998;58:3015-3020.

11 Saggiorato E, Cappia S, Giuli PD, et al. Galectin-3 as a presurgical immunodiagnostic marker of minimally invasive follicular thyroid carcinoma. J Clin Endocrinol Metab 2001;86:5152-5158.

$12 \mathrm{Xu}$ XC, El-Naggar AK, Lotan R. Differential expression of galectin-1 and galectin-3 in thyroid tumors: potential diagnostic implications. Am J Pathol 1995;147 :815-822.

13 Ryu S, Jimi S, Takebayashi S. Thyroid carcinoma distinctly expresses intracellular fibronectin in vivo. Cancer Lett 1997;121:189-193.

14 Sugenoya A, Usuda N, Adachi W, et al. Immunohistochemical studies on the localization of fibronectin in human thyroid neoplastic tissues. Endocrinol Jpn 1988;35:111-120.

15 Takano T, Matsuzuka F, Sumizaki H, et al. Rapid detection of specific messenger RNAs in thyroid carcinomas by reverse transcription-PCR with degenerate primers: specific expression of oncofetal fibronectin messenger RNA in papillary carcinoma. Cancer Res 1997;57:3792-3797.

16 Takano T, Miyauchi A, Yokozawa T, et al. Accurate and objective preoperative diagnosis of thyroid papillary carcinoma by reverse transcription-PCR detection of oncofetal fibronectin messenger RNA in fine-needle aspiration biopsies. Cancer Res 1998;58:4913-4917.

17 Fenner MH, Parrish JE, Boyd Y, et al. MSG1 (melanocyte specific gene 1): mapping to chromosome Xq13.1, genomic organization, and promoter analysis. Genomics 1998;51:401-407.

18 Shioda T, Fenner MH, Isselbacher KJ. Msg1, a novel melanocyte-specific gene, encodes a nuclear protein and is associated with pigmentation. Proc Natl Acad Sci USA 1996;93:12298-12303.

19 Raphael SJ, McKeown-Eyssen G, Asa SL. High-molecular-weight cytokeratin and cytokeratin-19 in the diagnosis of thyroid tumors. Mod Pathol 1994;7: 295-300.

20 Fonseca E, Nesland JM, Hoie J, et al. Pattern of expression of intermediate cytokeratin filaments in the thyroid gland: an immunohistochemical study of simple and stratified epithelial-type cytokeratin. Virchows Arch 1997;430:239-245.

21 Sahoo S, Hoda SA, DeLellis RA. Cytokeratin 19 immunoreactivity in the diagnosis of papillary thyroid carcinoma: a note of caution. Am J Clin Pathol 2001;116:696-702.

22 Beesley MF, McLaren KM. Cytokeratin 19 and galectin3 immunohistochemistry in the differential diagnosis of solitary thyroid nodule. Histopathology 2002;41: 236-243.

23 Cheung CC, Ezzat S, Freeman JL, et al. Immunohistochemical diagnosis of papillary thyroid carcinoma. Mod Pathol 2001;14:338-342.

24 Miettinen M, Karkkainen P. Differential reactivity of HBME1 and CD15 antibodies with benign and malignant thyroid tumors. Preferential reactivity with malignant tumors. Virchows Arch 1996;429:213-219.

25 Nikiforova MN, Lynch RA, Biddinger PW, et al. RAS point mutations and PAX8-PPAR gamma rearrangement in thyroid tumors: evidence for distinct molecular pathways in thyroid follicular carcinoma. J Clin Endocrinol Metab 2003;88:2318-2326.

26 Hoos A, Stojadinovic A, Singh B, et al. Clinical significance of molecular expression profiles of hürthle cell tumors of the thyroid gland analyzed via tissue microarrays. Am J Pathol 2002;160:175-183.

27 Rosai J, Caracangui M, DeLellis R. Follicular carcinoma. In: Rosai R (ed). Atlas of Tumor Pathology: Tumors of the Thyroid Gland, 3rd series. Armed Forces Institute of Pathology Press: Washington, DC, 1992, pp 49-64.

28 Williams ED. Guest editorial: two proposals regarding the terminology of thyroid tumors. Int J Surg Pathol 2000;8:181-183.

29 Bartolazzi A, Gasbarri A, Papotti M, et al. Application of an immunodiagnostic method for improving preoperative diagnosis of nodular thyroid lesions. Lancet 2001;357:1644-1650.

30 Kawachi K, Matsushita Y, Yonezawa S, et al. Galectin3 expression in various thyroid neoplasms and its possible role in metastasis formation. Hum Pathol 2000;31:428-433.

31 Martins L, Matsuo SE, Ebina KN, et al. Galectin-3 messenger RNA and proteins are expressed in benign thyroid tumors. J Clin Endocrinol Metab 2002;87: 4806-4810.

32 Niedziela M, Maceluch J, Korman E. Galectin-3 is not a universal marker of malignancy in thyroid nodular disease in children and adolescents. J Clin Endocrinol Metab 2002;87:4411-4415.

33 Basolo F, Molinaro E, Agate L, et al. RET protein expression has no prognostic impact on the long-term outcome of papillary thyroid carcinoma. Eur J Endocrinol 2001;145:599-604.

34 French CA, Alexander EK, Cibas ES, et al. Genetic and biological subgroups of low stage follicular thyroid cancer. Am J Pathol 2003;162:1053-1060.

35 Tomoda C, Kushima R, Takeuti E, et al. CD10 expression is useful in the diagnosis of follicular carcinoma and follicular variant of papillary thyroid carcinoma. Thyroid 2003;13:291-295.

36 Zhu Z, Gandhi M, Nikiforova MN, et al. Molecular profile and clinicopathological features of the follicular variant of papillary thyroid carcinoma. Am J Clin Pathol 2003;120:71-77. 